\title{
Percepción de comerciantes y compradores respecto a la presencia de perros y gatos vagabundos dentro de los mercados y las estrategias de control en el distrito de Los Olivos, Lima-Perú
}

\author{
Perception of sellers and buyers about the presence of stray dogs and cats in the \\ markets and their control strategies in Los Olivos district, Lima-Peru
}

\author{
Annghie Sandoval ${ }^{1}$, Daphne León ${ }^{2,3}$, Néstor Falcón ${ }^{2}$
}

\section{Resumen}

El objetivo del estudio fue evaluar la percepción de comerciantes y compradores acerca de la presencia de perros y gatos vagabundos dentro de los mercados y su estrategia de control en el distrito de Los Olivos en 2019. Se encuestó a 20 comerciantes y 20 compradores en cada uno de los 10 mercados seleccionados (total 400 encuestas). El 79.5 y $60.3 \%$ de ellos observaron perros y gatos vagabundos, respectivamente. El 59.2\% afirmaron que tenían propietarios. El 59.9\% de los comerciantes y el 36.2\% de los compradores indicaron que la presencia de perros en los mercados era principalmente debido a que los propietarios los llevaban. El 78.4\% opinó que representan un problema porque ensucian el ambiente $(66.9 \%)$, transmiten enfermedades $(35.6 \%)$ y contaminan los alimentos $(30.1 \%)$. Los padecimientos percibidos en los animales vagabundos eran la «desnutrición» $(31.3 \%)$, parasitosis $(24.3 \%)$ y accidentes (11\%). Según los encuestados, los encargados de enfrentar la presencia de estos animales en los mercados eran la municipalidad (37.7\%), los compradores (32.2\%) y la administración del mercado (20.9\%). La solución para este problema sería, a juicio de los encuestados, la adopción (43.3\%), la esteri-

\footnotetext{
${ }^{1}$ Grupo de Salud Pública en Veterinaria (SAPUVET-PERÚ), Facultad de Medicina Veterinaria y Zootecnia, Universidad Peruana Cayetano Heredia, Lima, Perú

${ }^{2}$ Laboratorio de Epidemiología y Salud Pública en Veterinaria, Facultad de Medicina Veterinaria y Zootecnia, Universidad Peruana Cayetano Heredia, Lima, Perú

${ }^{3}$ E-mail: daphne.leon@upch.pe; https://orcid.org/0000-0003-0346-4647
}

Recibido: 13 de octubre de 2020

Aceptado para publicación: 24 de marzo de 2021

Publicado: 23 de junio de 2021

CLos autores. Este artículo es publicado por la Rev Inv Vet Perú de la Facultad de Medicina Veterinaria, Universidad Nacional Mayor de San Marcos. Este es un artículo de acceso abierto, distribuido bajo los términos de la licencia Creative Commons Atribución 4.0 Internacional (CC BY 4.0) [https:// creativecommons.org/licenses/by/4.0/deed.es] que permite el uso, distribución y reproducción en cualquier medio, siempre que la obra original sea debidamente citada de su fuente original 
lización/castración (21.2\%), la concienciación en tenencia responsable de animales de compañía (13.2\%) y solo la esterilización de hembras (8.6\%). Los resultados muestran la necesidad de implementar un programa de sensibilización y cumplimiento de normas que prohíben la presencia de perros y gatos vagabundos en los mercados de Los Olivos.

Palabras clave: salud pública, mercados, zoonosis, canes vagabundos

\section{AbSTRACT}

The aim of this study was to evaluate the perception of sellers and buyers about the presence of stray dogs and cats within the markets and their control strategy in the Los Olivos district in 2019. In total, 20 sellers and 20 buyers were surveyed in each of the 10 selected markets (total 400 surveys). The results showed that 79.5 and $60.3 \%$ of the surveyed observed stray dogs and cats, respectively; $59.2 \%$ stated that they had owners; $59.9 \%$ of sellers and $36.2 \%$ of buyers indicated the presence of dogs in the markets was mainly due to the owners bringing them. Besides, $78.4 \%$ said that they represent a problem because they pollute the environment (66.9\%), transmit diseases $(35.6 \%)$ and contaminate food $(30.1 \%)$. The ailments perceived in stray animals were «malnutrition» $(31.3 \%)$, parasitosis $(24.3 \%)$ and accidents $(11 \%)$. According to them, the responsibility of dealing with the presence of these animals in the market rests in the municipality $(37.7 \%)$, buyers $(32.2 \%)$ and the market administration $(20.9 \%)$. The solution to this problem would be, in the opinion of those surveyed, adoption (43.3\%), spaying/neutering $(21.2 \%)$, awareness of responsible pet ownership (13.2\%) and only the sterilization of females $(8.6 \%)$. The results show the need to implement an awareness programme and compliance with regulations that prohibit the presence of stray dogs and cats in Los Olivos markets.

Key words: public health, markets, zoonoses, stray dogs

\section{INTRODUCCIÓN}

Se denomina perros vagabundos a los perros que no son controlados directamente por una persona y deambulan libremente, pudiendo ser de tres tipos: errantes con propietario, errantes sin propietario $\mathrm{y}$ asilvestrados (OIE, 2011). En el caso de los gatos, pueden encontrarse tres categorías: asilvestrados, vagabundos y errantes que poseen propietario (Lucano, 2018). La clasificación de los animales vagabundos es de importancia para definir su origen y plantear soluciones para su control (ICAM, 2007, 2011).
La condición socioeconómica influye en la crianza y el cuidado que se brinda a los perros y gatos. Las zonas de estrato socioeconómico bajo poseen mayor cantidad de animales vagabundos y con problemas que afectan su bienestar (Barbosa et al., 2018). En el distrito de Los Olivos, Lima, se estimó la presencia de $1411 \pm 643$ perros vagabundos en horario diurno y de $922 \pm 497$ en horario nocturno, observándose que la mayoría tenía un bajo índice de condición corporal (Ochoa et al., 2014). En Villa El Salvador, Lima, se encontró un promedio de 24.6 perros al interior de los mercados y 27.1 perros en los exteriores (Chávez et al., 2016). 
Estos animales encuentran alojamiento y alimento en los mercados, pero a su vez generan daños a la población. Pueden transmitir enfermedades zoonóticas (Platts-Mills et al., 2011; Miranda et al., 2014; Puerto y Tovar, 2016; Valeri, 2017), provocar accidentes por mordedura (Schvartzman y Pacín, 2005; Morales et al., 2011), desórdenes como peleas y juegos entre ellos, y contaminar el ambiente al dispersar la basura y al orinar o defecar en espacios públicos (Güttler, 2005; ICAM, 2007).

En Perú, la presencia de animales de compañía dentro de los mercados de abasto está prohibida con el objetivo de asegurar la calidad sanitaria de los alimentos y bebidas (Congreso de la República, 2001; Ministerio de Salud, 2003) y el bienestar de estos animales (Municipalidad Distrital de Los Olivos, 2016). Sin embargo, estas normas son usualmente ignoradas por desconocimiento o desinterés.

Para asegurar la sostenibilidad y aplicación de estas normas es necesario tener en cuenta las preocupaciones y opiniones de la comunidad, así como conocer la percepción sobre si los perros y gatos vagabundos son considerados como un problema (ICAM, 2007). Determinar el nivel de involucramiento de las personas y la importancia que le otorgan a este tema permitirá evaluar las estrategias y medidas de control que serían aceptadas por la población. Por ello, el objetivo del estudio fue evaluar la percepción de comerciantes y compradores acerca de la presencia de perros y gatos vagabundos dentro de los mercados y sus estrategias de control, tomando como base el caso del distrito de Los Olivos, Lima.

\section{Materiales y Métodos}

El estudio se desarrolló en mercados del distrito Los Olivos, Lima, Perú, en 2019. El desarrollo de la base de datos y el análisis estadístico se realizó en la Facultad de Medi- cina Veterinaria y Zootecnia de la Universidad Peruana Cayetano Heredia (FAVEZ$\mathrm{UPCH})$.

Se elaboró una encuesta que consideró 15 preguntas de alternativas múltiples y respuestas cortas, con información demográfica de la persona encuestada, percepción sobre la presencia de perros y gatos vagabundos en el mercado, los problemas que producían, las estrategias de control y las personas o instituciones responsables de enfrentar el problema. La encuesta fue validada por juicio de expertos.

El tamaño de muestra se determinó mediante la fórmula de comprobación de una proporción para poblaciones desconocidas, tomando en cuenta las siguientes restricciones: nivel de confianza del $95 \%$, proporción referencial para la variable personas que consideran que la presencia de perros y gatos vagabundos en los mercados era un problema del 50\% (valor utilizado cuando se desconoce información previa) y error máximo admisible de 5\%. El tamaño mínimo de muestra calculado fue de 385 encuestas.

La población objetivo fueron compradores y comerciantes, mayores de edad, que se encontraban en los mercados seleccionados para el estudio. Se realizaron 400 encuestas, la mitad dirigida a comerciantes y la otra mitad a compradores. Del total de mercados formales registrados por la Oficina de Desarrollo Económico de la Municipalidad de Los Olivos $(n=44)$, se seleccionaron 10 mercados que contaban con la mayor cantidad de puestos de venta. En cada mercado se entrevistó a 20 comerciantes y 20 compradores. Las encuestas fueron aplicadas por dos personas entrenadas para cumplir esta función. Se encuestaron 2 mercados por día en jornadas de 4 horas y el tiempo aproximado por cada encuesta fue de 5 minutos.

Se coordinó con las autoridades de los mercados seleccionados para la realización de las encuestas. Para aplicar las encuestas, 
el investigador abordó a los compradores y comerciantes al interior de los mercados y solicitó su participación voluntaria. Se consideró a cualquier persona que tenga más de 18 años sin ningún tipo de distinción. Antes de comenzar la encuesta, se le entregó la hoja informativa del estudio, la que detallaba los procedimientos, beneficios, la confidencialidad de la información brindada y los derechos como participante. Las preguntas de la encuesta fueron leídas y las respuestas marcadas en el instrumento de recolección de datos por el encuestador.

La información obtenida de las encuestas fue transferida a una base de datos en el programa Microsoft Excel y analizadas mediante estadística descriptiva (frecuencias absolutas y relativas) utilizando el programa estadístico Stata 13.0. La asociación entre la categoría del entrevistado (compradores y comerciantes) y las respuestas a las preguntas realizadas fue evaluada mediante la prueba de Chi cuadrado.

\section{Resultados}

Se encuestaron a 400 persona de los cuales 200 fueron compradores y 200 comerciantes. El 69.0\% (138) de los compradores y el $73.5 \%$ (147) de los comerciantes fueron mujeres.

El 79.5\% (318) y el 60.3\% (241) de los encuestados manifestó haber visto perros y gatos vagabundos en el interior de los mercados, respectivamente. Entre los compradores, $70.5 \%$ (141) indicó haber visto perros y $50 \%$ (100) haber visto gatos, y entre los comerciantes, $88.5 \%$ (177) indicó haber visto perros y $70.5 \%$ (141) indicó haber visto gatos. La proporción de personas que observaron perros o gatos fue significativamente mayor entre los comerciantes $(\mathrm{p}<0.05)$.

Las razones de la presencia de perros y gatos vagabundos manifestada entre los encuestados que indicaron haber visto perros o gatos se presentan en el Cuadro 1. Solo se encontró diferencia significativa para la razón «vienen con dueños» en el caso de perros, siendo esta proporción mayor para los comerciantes $(\mathrm{p}<0.05)$.

Entre los encuestados, la proporción de personas que opinaban que la presencia de los animales vagabundos representaba un problema fue de $71.8 \%(107 / 149)$ entre los compradores y de $83.7 \%(154 / 184)$ entre los comerciantes; encontrándose diferencia significativa entre ambos grupos $(\mathrm{p}<0.05)$. Entre los compradores, $54.4 \%$ (81/149) consideraba que estos animales tenían dueño y entre los comerciantes, 63\% (116/184) respondió de igual manera.

Ensuciar el ambiente $(218,66.9 \%)$, transmitir enfermedades $(116,35.6 \%)$ y contaminar alimentos $(98,30.1 \%)$ fueron los principales problemas que ocasionarían estos animales en el interior de los mercados, según la opinión de los encuestados que consideraron la presencia de perros y/o gatos vagabundos en los mercados como un problema (156 compradores y 170 comerciantes) (Cuadro 2). De otra parte, la desnutrición $(125,31.3 \%)$ y las parasitosis $(97,24.3 \%)$ fueron los padecimientos reconocidos con más frecuencia por los encuestados (Cuadro 3).

Entre los encuestados que consideraban como un problema la presencia de estos animales (incluyendo a los que vieron o no animales al interior de los mercados), consideraron que la responsabilidad para solucionar el problema residía en la municipalidad (123, $37.7 \%$ ) y en los propios clientes (105, 32.2\%), esto último, especialmente indicado por los comerciantes $(\mathrm{p}<0.05$; Cuadro 4$)$.

Las principales soluciones propuestas para enfrentar el problema fueron las adopciones $(141,43.3 \%)$ y el control reproductivo quirúrgico tanto en machos como hembras $(69,21.2 \%)$ (Cuadro 5), siendo esta última opinión significativamente mayor entre los compradores $(\mathrm{p}<0.05)$ 
Cuadro 1. Razones de la presencia de perros y gatos vagabundos, según compradores y comerciantes de 10 mercados de abasto del distrito de Los Olivos - Lima (2019)

\begin{tabular}{|c|c|c|c|c|c|c|c|c|}
\hline \multirow{3}{*}{ Razones } & \multicolumn{4}{|c|}{ Perro } & \multicolumn{4}{|c|}{ Gato } \\
\hline & \multicolumn{2}{|c|}{$\begin{array}{c}\text { Comprador } \\
(n=141)\end{array}$} & \multicolumn{2}{|c|}{$\begin{array}{l}\text { Comerciante } \\
(n=177)\end{array}$} & \multicolumn{2}{|c|}{$\begin{array}{l}\text { Comprador } \\
(n=100)\end{array}$} & \multicolumn{2}{|c|}{$\begin{array}{c}\text { Comerciante } \\
(n=141)\end{array}$} \\
\hline & $\mathrm{n}$ & $\% 1$ & n. & $\%{ }^{1}$ & $\mathrm{n}$ & $\%{ }^{1}$ & $\mathrm{n}$ & $\%{ }^{1}$ \\
\hline $\begin{array}{l}\text { Vienen con } \\
\text { dueño }\end{array}$ & 51 & $36.2^{\mathrm{a}}$ & 106 & $59.9^{\mathrm{b}}$ & 10 & 10.0 & 8 & 5.7 \\
\hline Buscan alimento & 40 & 28.4 & 35 & 19.8 & 32 & 32.0 & 45 & 31.9 \\
\hline Abandono & 40 & 28.4 & 38 & 21.5 & 39 & 39.0 & 51 & 36.2 \\
\hline $\begin{array}{l}\text { Descuido del } \\
\text { dueño }\end{array}$ & 11 & 7.8 & 12 & 6.8 & 7 & 7.0 & 6 & 4.3 \\
\hline Vagabundos & 5 & 3.5 & 9 & 5.1 & 7 & 7.0 & 19 & 13.5 \\
\hline Sobrepoblación & 3 & 2.1 &. & $\therefore$ & 2 & 2.0 & 3 & 2.1 \\
\hline $\begin{array}{l}\text { Falta de } \\
\text { vigilancia }\end{array}$ & 1 & 0.7 & 2 & 1.1 & 2 & 2.0 & 1 & 0.7 \\
\hline $\begin{array}{l}\text { Falta de } \\
\text { limpieza }\end{array}$ & 1 & 0.7 &. &. &.- &.- &.- &. \\
\hline $\begin{array}{l}\text { Control de } \\
\text { roedores }\end{array}$ &. &. &. &. & 2 & 2.0 & 10 & 7.1 \\
\hline Son del mercado &. &.- & .- &. & 3 & 3.0 & 3 & 2.1 \\
\hline
\end{tabular}

${ }^{1}$ Valor calculado en relación al número de compradores o comerciantes que observaron perros o gatos. Los encuestados podían ofrecer más de una respuesta

$a, b$ Letras diferentes indican que las proporciones dentro de filas y por especie animal son significativamente diferentes $(p<0.05)$

\section{Discusión}

La mayoría de encuestados que observaron perros y/o gatos vagabundos dentro de los mercados fueron comerciantes, lo cual podría deberse a su mayor permanencia en los locales. Así mismo, la especie más observada tanto por compradores como vendedores fueron los perros. La mayoría de los comerciantes que afirmaron haber visto perros vagos, indicaron que estos son llevados a los mercados por los propietarios (sean compra- dores o vendedores) como acompañantes o guardianes de los puestos. Tomando en consideración la definición de OIE (2011), estos animales corresponderían a los perros vagabundos con dueño. Llalla (2012) menciona que el perro es el animal de compañía más frecuente en las viviendas.

La búsqueda de alimento por perros y gatos es una razón frecuentemente mencionada por los encuestados. Al respecto, Chávez et al. (2016) encontraron más perros en los puestos de comercialización de carnes, aba- 
Cuadro 2. Opiniones de los encuestados acerca de los problemas que ocasionan los perros y gatos vagabundos en el interior de los mercados de abasto del distrito de Los Olivos - Lima (2019)

\begin{tabular}{|c|c|c|c|c|c|c|}
\hline \multirow[t]{2}{*}{ Opinión } & \multicolumn{2}{|c|}{$\begin{array}{l}\text { Comprador } \\
(n=156)\end{array}$} & \multicolumn{2}{|c|}{$\begin{array}{l}\text { Comerciante } \\
(n=170)\end{array}$} & \multicolumn{2}{|c|}{$\begin{array}{c}\text { Total } \\
(\mathrm{n}=326)\end{array}$} \\
\hline & $\mathrm{n}$ & $\% 1$ & $\mathrm{n}$ & $\%^{1}$ & $\mathrm{n}$ & $\%{ }^{1}$ \\
\hline Ensucian el ambiente & 99 & 63.5 & 119 & 70.0 & 218 & 66.9 \\
\hline $\begin{array}{l}\text { Transmiten } \\
\text { enfermedades }\end{array}$ & 61 & 39.1 & 55 & 32.4 & 116 & 35.6 \\
\hline $\begin{array}{l}\text { Contaminan } \\
\text { alimentos }\end{array}$ & 40 & 25.6 & 58 & 34.1 & 98 & 30.1 \\
\hline $\begin{array}{l}\text { Producen mordeduras } \\
\text { o arañazos }\end{array}$ & 27 & 17.3 & 24 & 14.1 & 51 & 15.6 \\
\hline Peleas entre animales & 14 & 9.0 & 15 & 8.8 & 29 & 8.9 \\
\hline $\begin{array}{l}\text { Rompen bolsas de } \\
\text { basura }\end{array}$ & 12 & 7.7 & 13 & 7.6 & 25 & 7.7 \\
\hline $\begin{array}{l}\text { Reproducción no } \\
\text { controlada }\end{array}$ & 2 & 1.3 & 1 & 0.6 & 3 & 0.9 \\
\hline $\begin{array}{l}\text { Mal aspecto e } \\
\text { incomodidad }\end{array}$ & - & - & 7 & 4.1 & 7 & 2.1 \\
\hline
\end{tabular}

${ }^{1}$ Valor calculado en relación al número de compradores o comerciantes que observaron perros o gatos. Los encuestados podían ofrecer más de una respuesta

rrotes y comida en los mercados del distrito de Villa El Salvador; debido a la posibilidad de que los comerciantes podrían entregar despojos y los comensales podrían entregar los restos de comida a los animales. Resultados similares fueron hallados por Veintimilla (2017) en un estudio demográfico de perros vagabundos en mercados en la ciudad de Quito, Ecuador. En el caso de los que observaron gatos, la razón predominante de la presencia en el mercado fue considerada al abandono de estos dentro o fuera del mercado. Los gatos vagabundos usualmente viven cerca a lugares donde pueden conseguir alimento, como es el caso de los mercados (ICAM, 2011).
La mayoría de los encuestados que observaron perros y/o gatos en los mercados, consideraron que la presencia de estos animales representa un problema. Sin embargo, este porcentaje fue mayor entre los comerciantes, posiblemente debido a que estos animales maltratan y ensucian su mercadería, volviéndola no apta para la venta. Además, causan incomodidad y dan un aspecto insalubre al lugar, provocando disminución en sus ventas y ganancias. Los encuestados mencionaron que los perros y gatos vagabundos ensucian el ambiente con heces y orina, transmiten ciertas enfermedades y contaminan los alimentos. Niklitschek (2016) en su estudio en Chile, encontró que las personas hacen 
Cuadro 3. Opiniones de los encuestados ${ }^{1}$ acerca de los padecimientos que sufren los perros y/o gatos vagabundos en el interior de los mercados de abasto del distrito de Los Olivos - Lima (2019)

\begin{tabular}{lcccccc}
\hline \multirow{2}{*}{ Opinión } & \multicolumn{2}{c}{$\begin{array}{c}\text { Comprador } \\
(\mathrm{n}=200)\end{array}$} & \multicolumn{2}{c}{$\begin{array}{c}\text { Comerciante } \\
(\mathrm{n}=200)\end{array}$} & \multicolumn{2}{c}{$\begin{array}{c}\text { Total } \\
(\mathrm{n}=400)\end{array}$} \\
\cline { 2 - 7 } & $\mathrm{n}$ & $\%$ & $\mathrm{n}$ & $\%$ & $\mathrm{n}$ & $\%$ \\
\hline Desnutrición & 59 & 29.5 & 66 & 33.0 & 125 & 31.3 \\
Parasitosis & 48 & 24.0 & 49 & 24.5 & 97 & 24.3 \\
Accidentes & 24 & 12.0 & 20 & 10.0 & 44 & 11.0 \\
Maltratos & 23 & 11.5 & 21 & 10.5 & 44 & 11.0 \\
Enfermedades & 20 & 10.0 & 11 & 5.5 & 31 & 7.8 \\
Abandono & 3 & 1.5 & 5 & 2.5 & 8 & 2.0 \\
Estrés & 1 & 0.5 &..- &.- & 1 & 0.3 \\
\hline
\end{tabular}

${ }^{1}$ Los encuestados podían ofrecer más de una respuesta

Cuadro 4. Opiniones de los encuestados ${ }^{1}$ acerca de quienes deberían de enfrentar el problema la presencia de perros y/o gatos vagabundos en el interior de los mercados de abasto del distrito de Los Olivos - Lima (2019)

\begin{tabular}{|c|c|c|c|c|c|c|}
\hline \multirow[t]{2}{*}{ Opinión } & \multicolumn{2}{|c|}{$\begin{array}{c}\text { Comprador } \\
(\mathrm{n}=200)\end{array}$} & \multicolumn{2}{|c|}{$\begin{array}{l}\text { Comerciante } \\
(n=200)\end{array}$} & \multicolumn{2}{|c|}{$\begin{array}{c}\text { Total } \\
(n=400)\end{array}$} \\
\hline & $\mathrm{n}$ & $\%$ & $\mathrm{n}$ & $\%$ & $\mathrm{n}$ & $\%$ \\
\hline Municipalidad & 64 & 41.0 & 59 & 34.7 & 123 & 37.7 \\
\hline Cliente mercado & 40 & $25.6^{\mathrm{a}}$ & 65 & $38.2^{\mathrm{b}}$ & 105 & 32.2 \\
\hline $\begin{array}{l}\text { Administración } \\
\text { mercado }\end{array}$ & 37 & 23.7 & 31 & 18.2 & 68 & 20.9 \\
\hline Propietario puesto & 13 & 8.3 & 14 & 8.2 & 27 & 8.3 \\
\hline Ministerio de salud & 10 & 6.4 & 8 & 4.7 & 18 & 5.5 \\
\hline Centro de rescate & 2 & 1.3 & 4 & 2.4 & 6 & 1.8 \\
\hline No hay responsables & 1 & 0.6 & $\therefore$ & $\therefore$ & 1 & 0.3 \\
\hline Veterinarios & 1 & 0.6 &. & $\therefore$ & 1 & 0.3 \\
\hline $\begin{array}{l}\text { Beneficencia } \\
\text { voluntaria }\end{array}$ &. &. & 1 & 0.6 & 1 & 0.3 \\
\hline
\end{tabular}

${ }^{1}$ Los encuestados podían ofrecer más de una respuesta

a.b Letras diferentes indican que las proporciones en cada fila son significativamente diferentes $(p<0.05)$ 
Cuadro 5. Opiniones de los encuestados ${ }^{1}$ acerca de las soluciones para enfrentar el problema la presencia de perros y/o gatos vagabundos en el interior de los mercados de abasto del distrito de Los Olivos - Lima (2019)

\begin{tabular}{|c|c|c|c|c|c|c|}
\hline \multirow[t]{2}{*}{ Opinión } & \multicolumn{2}{|c|}{$\begin{array}{l}\text { Comprador } \\
(n=156)\end{array}$} & \multicolumn{2}{|c|}{$\begin{array}{c}\text { Comerciante } \\
\quad(n=170)\end{array}$} & \multicolumn{2}{|c|}{$\begin{array}{c}\text { Total } \\
(n=326)\end{array}$} \\
\hline & $\mathrm{n}$ & $\%$ & $\mathrm{n}$ & $\%$ & $\mathrm{n}$ & $\%$ \\
\hline Adopción & 66 & 42.3 & 75 & 44.1 & 141 & 43.3 \\
\hline $\begin{array}{l}\text { Esterilización hembras/ } \\
\text { castración macho }\end{array}$ & 42 & $26.9^{\mathrm{a}}$ & 27 & $15.9^{\mathrm{b}}$ & 69 & 21.2 \\
\hline $\begin{array}{l}\text { Concienciación en } \\
\text { TRAC }^{2}\end{array}$ & 18 & 11.5 & 25 & 14.7 & 43 & 13.2 \\
\hline $\begin{array}{l}\text { Esterilización de } \\
\text { hembras }\end{array}$ & 13 & 8.3 & 15 & 8.8 & 28 & 8.6 \\
\hline $\begin{array}{l}\text { Más vigilancia en } \\
\text { mercados }\end{array}$ & 12 & 7.7 & 20 & 11.8 & 32 & 9.8 \\
\hline Eutanasia & 10 & 6.4 & 8 & 4.7 & 18 & 5.5 \\
\hline Castración de machos & 6 & 3.8 & 7 & 4.1 & 13 & 4.0 \\
\hline $\begin{array}{l}\text { Sanciones de } \\
\text { propietarios }\end{array}$ & 5 & 3.2 & 10 & 5.9 & 15 & 4.6 \\
\hline No criar animales & 2 & 1.3 & 2 & 1.2 & 4 & 1.2 \\
\hline $\begin{array}{l}\text { Colocar comederos en } \\
\text { exteriores }\end{array}$ & 2 & 1.3 & 2 & 1.2 & 4 & 1.2 \\
\hline Limpieza de mercado & 1 & 0.6 & 1 & 0.6 & 2 & 0.6 \\
\hline Crear ordenanzas & 1 & 0.6 & 1 & 0.6 & 2 & 0.6 \\
\hline
\end{tabular}

${ }^{1}$ Los encuestados podían ofrecer más de una respuesta

${ }^{2}$ Tenencia responsable de animales de compañía

a.b Letras diferentes indican que las proporciones en cada fila son significativamente diferentes $(p<0.05)$

mayor referencia a la suciedad causada por estos animales $(90.5 \%)$, quedando en cuarto lugar la transmisión de enfermedades $(85.1 \%)$.

Según la percepción de los encuestados, la desnutrición y parasitosis son las principales consecuencias que sufren los perros y gatos vagabundos en los mercados, en similitud con la percepción que tuvieron los estudiantes de medicina veterinaria en el estudio de Vizquerra et al. (2017). Probablemente, los encuestados consideran como «desnutrición» a la baja condición corporal en los animales vagabundos, como se observó en los estudios de Ochoa et al. (2014) y Chávez et al. (2016). La apariencia de «desnutridos» puede ser por el padecimiento de enfermedades crónicas u otras que, sumado al limitado alimento de calidad al que tienen acceso los animales, podría ofrecer esa percepción a los encuestados. 
La percepción de la parasitosis es apoyada por la publicación de Náquira (2010), donde menciona que dos de las zoonosis parasitarias más importantes en el Perú son la toxocariasis y la equinococosis quística. Una fuente de infección importante para la toxocariasis son los espacios públicos contaminados. Así, en estudios de los suelos de parques de Lima Metropolitana y Callao se halló que $34.3 \%$ de los parques del Cono Norte estaban contaminados con huevos de Toxocara sp, siendo el distrito de Los Olivos el más contaminado ( $44 \%$ de parques positivos) (Chávez et al., 2002).

El consumo de carnes y/o vísceras crudas (alimentos disponibles en los mercados) pueden generar la transmisión de ciertas zoonosis. La sierra central de Perú es endémica a equinococosis (Guerra y Ramírez, 2015). El ganado de estas zonas muchas veces es trasladado hasta Lima para comercializar su carne y vísceras en diversos mercados, y las fallas en el control de inocuidad podrían originar fuentes de contagio de Echinococcus granulosus. En el cono Norte, que incluye a Los Olivos, se reportó una prevalencia de equinococosis en perros de al menos 1\% (Chuquisana et al., 2014). En el caso de la toxoplasmosis, los gatos pueden infectarse con la ingesta de quistes tisulares (bradizoitos) al consumir carne de aves, roedores, bovinos, porcinos u ovinos infectados con Toxoplasma gondii (Acha y Szyfres, 2001).

Los encuestados mencionaron también que los animales sufrían accidentes automovilísticos al intentar ingresar o salir de los mercados. En Argentina, el 13\% de los accidentes viales eran causados por animales sueltos, donde se incluye a los perros vagabundos (Arata y Reátegui, 2016). De otra parte, el maltrato animal fue considerado como factor para el sufrimiento de los animales. Lasso (2016) menciona que esta situación podría alterar su comportamiento tornándose agresivos con las personas y otros animales, generando accidentes por mordedura o arañazos.
Los comerciantes y compradores opinaron que la municipalidad del distrito debería encargarse de enfrentar el problema de animales vagabundos en los mercados. Asimismo, los mismos compradores y la administración del mercado fueron la segunda y tercera opción más votadas, respectivamente. Según Vizquerra et al. (2017), la mayoría de los encuestados en su estudio consideró a la municipalidad como la responsable de este problema. En cambio, Niklitschek (2016), en la Región de los Lagos, Chile, encontraron que la responsabilidad fue atribuida en primera instancia a los propietarios de los perros, seguida de la municipalidad y del servicio de salud. De otra parte, la OIE (2011) asigna a la administración del mercado la responsabilidad de coordinar con la municipalidad para desarrollar y ejecutar planes que garanticen la calidad de los productos de consumo humano ofrecidos en los mercados.

El Ministerio de Salud fue poco mencionado por los encuestados, pese a que este, juntamente con las municipalidades de los distritos, son las entidades encargadas de promover los programas de salud preventiva, de tenencia responsable de animales de compañía (TRAC) y educación sanitaria como lo indican la Ley N. ${ }^{\circ} 27596$, Ley que Regula el Régimen Jurídico de Canes (2001) y su Reglamento aprobado por Decreto Supremo $\mathrm{N}^{\circ} 006-2002-\mathrm{SA}$ (MINSA, 2002).

Como solución principal para enfrentar el problema, la mayoría de los encuestados eligió la adopción (43.3\%). Sin embargo, la OIE (2011) indica que el número de perros disponibles puede llegar a superar la cantidad de adoptantes. La esterilización de hembras y machos fue la segunda opinión más mencionada, especialmente por los compradores, la cual, en teoría, sería la mejor opción para regular el número de perros y gatos, ya que disminuiría la cifra de cachorros que no encontrarán un hogar (Jaramillo, 2016). Lamentablemente hay una percepción negativa acerca de la esterilización. Arata y Reategui (2016) encontraron que el 55\% de la población encuestada en el distrito de Surquillo, 
Lima, estaba en contra de este método ya que lo consideraron cruel. Estas posturas se reflejan en la cantidad de perros que no están bajo control reproductivo, como en los distritos de San Martín de Porres (Arauco et al., 2014) y Bellavista-Callao (Harada et al., 2019), donde más del $80 \%$ de los perros no estaban castrados o esterilizados.

Por otro lado, la esterilización de hembras ha tenido mayor aceptación que la castración de los machos, a pesar de que esta última es más sencilla y el tiempo de recuperación de los animales es más corto (Edwards y Chávez, 2012; Spahr, 2019). Es probable que esta diferencia se asocie al hecho de que las crías generalmente quedan a responsabilidad del propietario de las hembras generando gastos extras, preocupación e incomodidad, por lo que la esterilización sería la alternativa para evitar estos problemas. La preferencia por esterilizar hembras se encuentra también en otros estudios como el de Rojas et al., 2019) en el distrito de Los Olivos, Lima, donde el $90.7 \%$ de perros y el $89 \%$ de gatos esterilizados en la Clínica Veterinaria Municipal fueron hembras.

La concienciación en TRAC fue una opción considerada con menor preferencia, pero que es importante a mediano y a largo plazo. La TRAC consiste en el cuidado adecuado de los perros y gatos, garantizando su bienestar y donde los propietarios deben minimizar el riesgo que su animal de compañía pueda representar para la sociedad (ICAM, 2007).

Por otro lado, la eutanasia fue una opción de escasa preferencia. La OIE (2011) menciona que la eutanasia por sí sola no es una medida eficaz de control, y solo debería utilizarse de manera compasiva y acompañada con otros métodos de control reproductivo para obtener un resultado eficaz a largo plazo. ICAM (2007) afirma que esta podría ser una buena opción para animales heridos, enfermos o con comportamien- tos sin solución, así como para animales que pasaron un largo tiempo en perreras sin conseguir adopción.

La promulgación de ordenanzas y sanciones a propietarios fueron opciones poco mencionadas, pero dieron evidencia de la desinformación que poseen las personas acerca de los reglamentos ya establecidos en este distrito. A nivel nacional existe la Ley N. ${ }^{\circ}$ 27596, Ley que Regula el Régimen Jurídico de Canes. También se debe tener en cuenta el Reglamento Sanitario de Funcionamiento de Mercados de Abasto y la Ordenanza Municipal N. ${ }^{\circ} 435-\mathrm{CDLO}$, que prohíbe el ingreso de animales a lugares públicos sin compañía de una persona responsable. La presencia de perros y gatos vagabundos en lugares públicos no autorizados, como son los mercados, refleja el incumplimiento de las anteriores normas, revelando una tenencia irresponsable de animales de compañía.

El problema de los perros y gatos vagabundos presenta una serie de causas donde la actitud de las personas frente a este problema es la principal determinante (Totton $e t$ al., 2010). El desconocimiento de la población respecto al impacto en la salud pública influye en la desatención del problema. Es por ello que estos programas deben de complementarse con actividades educativas en TRAC y enfermedades zoonóticas; además del manejo de residuos y saneamiento ambiental (Cadena, 2013), lo que ha de permitir un manejo integral de la problemática y una mayor probabilidad de éxito.

\section{Conclusiones}

- $\quad$ El 79.5\% del total de encuestados observó perros vagabundos y el $60.3 \%$ gatos vagabundos en el interior de los mercados del distrito de Los Olivos, Lima.

- La mayoría de los compradores y comerciantes opinan que los perros y/o gatos vagabundos tienen propietario. 
- La mayoría opina que la presencia de perros y/o gatos dentro de los mercados representa un problema porque ensucian el ambiente, transmiten enfermedades y contaminan los alimentos.

- La «desnutrición» y la parasitosis fueron percibidos como los padecimientos predominantes en perros y gatos vagabundos.

- Los encuestados mencionan a la municipalidad y a los compradores como los principales responsables de enfrentar el problema de animales vagabundos en los mercados

- Las mejores estrategias para el control de perros y/o gatos vagabundos serían las adopciones, esterilizaciones/castraciones y concienciación en tenencia responsable de animales de compañía.

\section{Literatura Cittada}

1. Acha P, Szyfres B. 2001. Salmonelosis. En: Zoonosis y enfermedades transmisibles comunes al hombre y a los animales. Vol. 3. Parasitosis. $3^{\circ}$ ed. Washington: Organización Panamericana de la Salud. 90 p.

2. Arata C, Reategui G 2016. Programa de control de la población canina en el distrito de Surquillo Lima, Perú. Tesis de Maestría. Lima, Perú: Univ. Peruana de Ciencias Aplicadas. 166 p.

3. Arauco D, Urbina B, León D, Falcón N. 2014. Indicadores demográficos y estimación de la población de canes con dueño en el distrito de San Martín de Porres, Lima-Perú. Salud Tecnol Vet 2: 83-92.

4. Barbosa Costa G, Gilbert A, Monroe $B$, Blanton J, Ngam Ngam S, Recuenco $S$, Wallace R. 2018. The influence of poverty and rabies knowledge on healthcare seeking behaviors and dog ownership, Cameroon. Plos One 13: e0197330. doi: 10.1371/journal.pone. 0197330
5. Cadena G 2013. Estudio para la estimación de la población de perros callejeros en mercados municipales del distrito Metropolitano de Quito. DMQ. Tesis de Médico Veterinario. Quito, Ecuador: Univ. San Francisco De Quito. 139 p.

6. Chávez A, Casas E, Serrano M, Cajas J, Velarde J, La Rosa V, López J. 2002. Riesgo de contraer enfermedades parasitarias en los parques públicos de Lima y Callao. Rev Inv Vet Perú 13: 84-91.

7. Chávez C, Falcón N, León D, Sánchez D. 2016. Canes Vagabundos en el interior y alrededores de mercados formales de Villa El Salvador, Lima, Perú. Rev Inv Vet Perú 27: 176-182. doi: 10.15381/ rivep.v27i1.11456

8. Chuquisana J, Chávez A, Casas E. 2014. Determinación de Echinococcus granulosus en perros del cono Norte de Lima. Rev Inv Vet Perú 11: 24-29. doi: 10.15381/rivep.v11i2.7055

9. Congreso de la República. 2001. Ley $N^{\circ}$ 27596. Ley que Regula el Régimen Jurídico de Canes. El Peruano. p 213876 213878.

10. Edwards C, Chávez G 2012. ¿Qué es preferible tener un macho o una hembra?. Murmullos Filosóficos 2: 36-40.

11. Guerra L, Ramírez M. 2015. Hidatidosis humana en el Perú. Apunt Cienc Soc 5: 94-101.

12. Güttler V. 2005. Análisis de algunas características de la población canina relacionadas con mordeduras e hidatidosis humana en la provincia de Valdivia. Tesis de Médico Veterinario. Chile: Univ. Austral de Chile. 64 p.

13. Harada C, León D, Gamarra N, Falcón $N$. 2019. Indicadores demográficos y estimación de la población de canes en el distrito de Bellavista, Callao - Perú. Salud Tecnol Vet 1:27-32.

14. [ICAM] Coalición Internacional para el Manejo de Animales de Compañía. 2007. Guía para el manejo humanitario de poblaciones caninas. $24 \mathrm{p}$. [Internet]. Disponible en: https://asanda.org/docu- 
mentos/animales-domesticos/Guia ManejoHumanitarioPoblacionesCaninas.pdf

15. [ICAM] Coalición Internacional para el Manejo de Animales de Compañía. 2011. Human cat population management guidance. 38 p. [Internet]. Disponible en: https://www.icam-coalition.org/ wp-content/uploads/2017/03/Humanecat-population-English.pdf

16. Jaramillo R. 2016. Adopción y tenencia responsable de mascotas. Tesis de Periodista. Santiago de Chile: Univ. de Chile. $65 \mathrm{p}$.

17. Lasso M. 2016. Amistad callejera. Tesis Doctoral. Colombia: Corporación Universitaria Minuto de Dios. $70 \mathrm{p}$.

18. Llalla H. 2012. Caracterización de la tenencia de mascotas y evaluación de conocimientos, actitudes y prácticas asociadas a riesgos de zoonosis y accidentes entre escolares de educación secundaria en tres distritos de Lima Metropolitana. Tesis de Maestría. Lima: Univ. Peruana Cayetano Heredia. 83 p.

19. Lucano H. 2018. Book review: Agnès Dufau. Estatuto jurídico del gato callejero en España, Francia y Reino Unido. Valencia, España: Tirant lo Blanch. 109 p.

20. Ministerio de Salud. 2002. Aprueban Reglamento de la Ley que regula el Régimen Jurídico de Canes. Decreto Supremo $\mathrm{N}^{\mathrm{o}}$ 006-2002-SA. El Peruano. $\mathrm{p}$ 225240-225244.

21. Ministerio de Salud. 2003. Reglamento Sanitario de Funcionamiento de Mercados de Abasto. Resolución Ministerial $\mathrm{N}^{\mathrm{o}}$ 282-2003-SA/DM. El Peruano. $\mathrm{p}$ 246762-246778.

22. Miranda E, Candela J, Alvarado J, Esquivel M, Farfán S. 2014. Infección por Bartonella henselae como presentación de fiebre de origen desconocida en niños peruanos. Acta Med Per 31: 74-78.

23. Morales C, Falcón N, Hernández H, Fernández C. 2011. Accidentes por mordedura canina, casos registrados en un hospital de niños de Lima, Perú 19952009. Rev Perú Med Exp Salud Pública 28: 639-642.
24. Municipalidad Distrital de Los Olivos. 2016. Ordenanza que aprueba el reglamento de cumplimiento y aplicación de la Ley N 30407 - Ley de protección y bienestar animal, en el distrito de Los Olivos. Ordenanza Municipal $\mathrm{N}^{\circ} 435-$ CDLO. p 30.

25. Náquira C. 2010. Las zoonosis parasitarias: problema de salud pública en el Perú. Rev Peru Med Exp Salud Pública 27: 494-497.

26. Niklitschek C. 2016. Percepción del problema de perros en la vía pública y las soluciones propuestas, Frutillar, Región de los Lagos, Chile. Tesis de Médico Veterinario. Chile: Univ. Austral de Chile. 29 p.

27. Ochoa Y, Falcón N, Zuazo J, Guevara B. 2014. Estimación de la población de perros callejeros en el distrito de Los Olivos, Lima, Perú. Rev Inv Vet Perú 25: 366-373. doi: 10.15381/rivep.v25i3.10114

28. [OIE] Organización Mundial para la Salud Animal. 2011. Código Sanitario para los Animales Terrestres. Cap 7.7. El control de las poblaciones de perros vagabundos. [Internet]. Disponible en: http://www.oie.int/fileadmin/Home/esp/ Health_standards/tahc/2011/es_chapitre_1.7.7.pdf

29. Platts-Mills J, LaRochelle P, Campos K, Vinetz J, Gotuzzo E, Ricaldi J. 2011. Seroprevalencia de leptospirosis en Puente Piedra, Lima en el año 2006. Rev Peru Med Exp Salud Pública 28: 273-276.

30. Puerto C, Tovar S. 2016. Infección al sistema nervioso por Toxocara canis en Hospital Escuela Universitario, Honduras. Rev Fac Cienc Méd 13: 47-51.

31. Rojas P, León D, Falcón N. 2019. Características de los perros y gatos bajo control reproductivo quirúrgico registrados en la Municipalidad de Los Olivos, Lima, Perú. Periodo 2015-2016. Rev Inv Vet Perú 30: 818-827. doi: 10.15381/ rivep.v30i2.16093 
32. Schvartzman S, Pacín M. 2005. Lesiones por mordedura de perro en niños. Arch Argent Pediatr 103: 389-395.

33. Spahr CS. 2019. Castración del perro macho: una cuestión social. Área bromatología, Municipalidad de Sampacho (CBA). Tesis de Licenciatura en Publicidad. Argentina: Univ. Siglo $21.155 \mathrm{p}$.

34. Totton S, Wandeler A, Zinsstag J, Bauch C, Ribble C, Rosatte $R$, et al. 2010. Stray dog population demographics in Jodhpur, India following a population control/rabies vaccination program. Prev Vet Med 97: 51-57. doi: 10.1016/j.prevetmed.2010.07.009
35. Valeri R. 2017. Case of chronic recycling toxocararosis caused by Toxocara cati. Pediatr Infect Dis 2: 49. doi: 10.21767/2573-0282.100049

36. Veintimilla T. 2017. Estudio demográfico de perros callejeros en los mercados del noroeste y noreste de la ciudad de Quito. Tesis de Médico Veterinario Zootecnista. Quito: Univ. de las Américas. $60 \mathrm{p}$.

37. Vizquerra M, San Martín F, Carbajal I, Falcón N. 2017. Percepción de los estudiantes de medicina veterinaria acerca de los perros vagabundos y sus estrategias de control en Lima, Perú. Rev Inv Vet Perú 28: 1029-1038. doi: 10.15381/rivep.v28i4.13877 